\title{
The jaw is a second-class lever in Pedetes capensis (Rodentia: Pedetidae)
}

\author{
Philip G Cox ${ }^{\text {Corresp. } 1,2}$ \\ 1 Department of Archaeology, University of York, York, UK \\ 2 Hull York Medical School, University of York, York, UK \\ Corresponding Author: Philip G Cox \\ Email address: philip.cox@hyms.ac.uk
}

The mammalian jaw is often modelled as a third-class lever for the purposes of biomechanical analyses, owing to the position of the resultant muscle force between the jaw joint and the teeth. However, it has been proposed that in some rodents the jaws operate as a second-class lever during distal molar bites, owing to the rostral position of the masticatory musculature. In particular, the infraorbital portion of the zygomaticomandibularis (IOZM) has been suggested to be of major importance in converting the masticatory system from a third-class to a second-class lever. The presence of the IOZM is diagnostic of the hystricomorph rodents, and is particularly well-developed in Pedetes capensis, the South African springhare. In this study, finite element analysis (FEA) was used to assess the lever mechanics of the springhare masticatory system, and to determine the function of the IOZM. An FE model of the skull of $P$. capensis was constructed and loaded with all masticatory muscles, and then solved for biting at each tooth in turn. Further load cases were created in which each masticatory muscle was removed in turn. The analyses showed that the mechanical advantage of the springhare jaws was above one at all molar bites and very close to one during the premolar bite. Removing the IOZM or masseter caused a drop in mechanical advantage at all bites, but affected strain patterns and cranial deformation very little. Removing the ZM had only a small effect on mechanical advantage, but produced a substantial reduction in strain and deformation across the skull. It was concluded that the masticatory system of $P$. capensis acts as a second class lever during bites along almost the entire cheek tooth row. The IOZM is clearly a major contributor to this effect, but the masseter also has a part to play. The benefit of the IOZM is that it adds force without substantially contributing to strain or deformation of the skull. This may help explain why the hystricomorphous morphology has evolved multiple times independently within Rodentia. 
1 The jaw is a second-class lever in Pedetes capensis (Rodentia: Pedetidae)

2

3 Philip G. Cox ${ }^{1,2}$

$4{ }^{1}$ Department of Archaeology, University of York, York, YO10 5DD, UK

$5{ }^{2}$ Hull York Medical School, University of York, York, YO10 5DD, UK

6

7 Corresponding author:

8 Philip G. Cox

9 Email: philip.cox@hyms.ac.uk 
32 ABSTRACT

33 The mammalian jaw is often modelled as a third-class lever for the purposes of biomechanical analyses, owing to the position of the resultant muscle force between the jaw joint and the teeth. However, it has been proposed that in some rodents the jaws operate as a second-class lever during distal molar bites, owing to the rostral position of the masticatory musculature. In particular, the infraorbital portion of the zygomatico-mandibularis (IOZM) has been suggested to be of major importance in converting the masticatory system from a third-class to a second-class lever. The presence of the IOZM is diagnostic of the hystricomorph rodents, and is particularly well-developed in Pedetes capensis, the South African springhare. In this study, finite element analysis (FEA) was used to assess the lever mechanics of the springhare masticatory system, and to determine the function of the IOZM. An FE model of the skull of $P$. capensis was constructed and loaded with all masticatory muscles, and then solved for biting at each tooth in turn. Further load cases were created in which each masticatory muscle was removed in turn. The analyses showed that the mechanical advantage of the springhare jaws was above one at all molar bites and very close to one during the premolar bite. Removing the IOZM or masseter caused a drop in mechanical advantage at all bites, but affected strain patterns and cranial deformation very little. Removing the ZM had only a small effect on mechanical advantage, but produced a substantial reduction in strain and deformation across the skull. It was concluded that the masticatory system of $P$. capensis acts as a second class lever during bites along almost the entire cheek tooth row. The IOZM is clearly a major contributor to this effect, but the masseter also has a part to play. The benefit of the IOZM is that it adds force without substantially contributing to strain or deformation of the skull. This may help explain why the hystricomorphous morphology has evolved multiple times independently within Rodentia. 


\section{INTRODUCTION}

The mammalian jaw is frequently treated as a lever for the purposes of biomechanical analysis (e.g. Crompton, 1963; Bramble, 1978; Greaves, 1978, 1982, 2000; Gingerich, 1979; Thomason, 1991; Satoh, 1998; 1999; Spencer, 1998, 1999; Satoh \& Iwaku, 2006, 2009; Davis et al., 2010; Druzinsky, 2010; Cornette et al., 2012; Becerra et al., 2013; Santana, 2015). More specifically, it is frequently considered to be a third-class lever i.e. one in which the input force sits between the fulcrum and the output force (Kerr, 2010). In mammals, the resultant masticatory muscle force (the input force) is usually situated between the jaw joint (fulcrum) and the biting tooth (output force) and thus the comparison with a third-class lever is generally accurate. The advantage of positioning muscle force posterior to the teeth is that relatively wide gapes can be achieved and high tensile forces at the temporo-mandibular joint, which could lead to dislocation of the jaws, are avoided (Greaves, 2000, 2012). However, the trade-off is that the mechanical advantage of a third-class lever is always less than one - that is, the output bite force will always be less than the effective muscle force.

It has occasionally been proposed that mammalian jaws do not always operate as third-class levers (Davis, 1955; Turnbull, 1970), and can in certain circumstances act as second-class levers with the output force between fulcrum and input force. In his classic work on the mammalian masticatory system, Turnbull (1970) suggested that the relative size and position of the masseter in many rodents (and a few ungulates) can shift the resultant of the masticatory musculature anterior to the distal cheek teeth, converting the masticatory system into a second-class lever during distal molar biting. Such an effect has even been claimed to occur in humans, with the jaw operating as a second-class lever during bites on the second and third molars (Mansour \& Reynik, 1975). Alternatively, other authors have argued that although some parts of the muscle mass attach far forward on the jaws in rodents, the resultant muscle force is still located towards the posterior end of the jaw (Greaves, 2012).

In rodents, one muscle in particular has been identified as contributing to the jaw operating as a second-class lever. The infraorbital portion of the zygomatico-mandibularis (IOZM) is an anterior expansion of the deepest layer of the masseter, the zygomatico-mandibularis (ZM), which passes through the enlarged infraorbital foramen to take its origin on the lateral surface of 
94 the rostrum. The IOZM, also referred to as the maxillo-mandibularis (Becht, 1953; Turnbull, 95 1970) or medial masseter (Wood, 1965; Woods, 1972), is the one of the defining characters of hystricomorph rodents, but is also present in a somewhat smaller form in myomorphs, where it is found in combination with a rostral expansion of the deep masseter (Wood, 1965; Cox \& Jeffery, 2011). Given its rostral origin on the skull and its mandibular insertion at the level of the premolar, Becht (1953) believed the function of the IOZM was to convert the jaw from a thirdclass lever to a second-class lever during molar biting.

This study seeks to understand the lever mechanics of the skull in the South African springhare, Pedetes capensis - a rodent species in which the IOZM is notably well-developed (Offermans \& De Vree, 1989). P. capensis is a nocturnal, bipedal, saltatorial rodent that inhabits arid and semiarid areas of southern Africa (Peinke \& Brown, 2003). It is large for a rodent (3-4 kg) and feeds principally on grasses, especially the rhizomes of Cynodon dactylon and the tubers of Cyperus esculentus (Peinke \& Brown, 2006). P. capensis and its sister-species $P$. surdaster are the only two extant members of the Pedetidae (Wilson \& Reeder, 2005), a family which molecular analyses place as sister-group to the Anomaluridae (scaly-tailed flying squirrels) in the Anomaluromorpha, which itself is part of the mouse-related clade (Fabre et al., 2012). Given the presence of the IOZM muscle, the pedetids (and anomalurids) have been identified as being hystricomorphous (Wood, 1965). However, the hystricomorphy seen in the Anomaluromorpha has evolved independently from that seen in three other groups of rodents: the Ctenohystrica, the Dipodidae, and some members of the Gliridae (Hautier, Cox \& Lebrun, 2015). Thus, the function of the IOZM is of prime interest to understanding the evolution of the rodents - why has this muscle arisen independently so many times throughout rodents?

The aim of this study is to model the masticatory system of $P$. capensis to determine if it functions as a second or third-class lever, and to assess the function of the masticatory muscles, particularly the IOZM. There are two specific hypotheses that will be tested:

1. It is hypothesised that a model of the skull of $P$. capensis will demonstrate the masticatory system operating as a second-class lever along most of the molar tooth row. This is expected based on previous dissection work by Offermans and De Vree (1989) who showed that a great deal of the masticatory musculature is situated alongside or 
anterior to the cheek teeth. The masticatory system will be determined to be a secondclass lever when the bite force exceeds the effective muscle force, i.e. when the mechanical advantage is greater than one, and when the reaction force at the temporomandibular joint is negative.

2. It is hypothesised that the IOZM muscle has a major role in converting the masticatory system from a third to a second-class lever in $P$. capensis. This hypothesis was previously proposed by Becht (1953) and is also expected owing to the large size and rostral position of the IOZM (Offermans \& De Vree, 1989, 1993). The function of the IOZM will be determined by virtual ablation analyses i.e. removing it and other muscles from the models to elucidate the effect on the biomechanical performance of the system, as determined by mechanical advantage, principal strains and the overall deformation of the skull during biting.

Previous studies of the lever mechanics of the mammalian masticatory system have tended to focus on the mandible (Greaves, 1978; 1982, 2000; Spencer, 1998, 1999), owing to its relatively simple shape and because its function is largely limited to feeding. However, in this study, the skull will be analysed, because of the interest in the IOZM, which is a particularly unusual fanshaped and convergent muscle, originating on the rostrum. To address the hypotheses and to study the function of the springhare skull during biting, finite element analysis (FEA) will be employed. FEA is an engineering technique for predicting stress, strain and deformation in an object during loading (Rayfield, 2007), and is now frequently applied to reconstructions of skulls and other skeletal elements in order to analyse vertebrate biomechanics (e.g. Richmond et al., 2005; Kupczik et al., 2007; Dumont et al., 2011; Ross et al., 2011; Cox et al., 2012; Cox, Kirkham \& Herrel, 2013; O’Hare et al., 2013; Porro et al., 2013; Figueirido et al., 2014; Cuff, Bright \& Rayfield, 2015; Sharp, 2015; McIntosh \& Cox, 2016; McCabe et al., 2017; Tsouknidas et al., 2017). As well as simulating stress and strain distributions, FEA is also able to predict reaction forces, and so will be used here to estimate bite force, jaw joint reaction force and mechanical advantage. Although these metrics could in theory be estimated via simple 2D lever models, it has been shown that such simplification leads to inaccuracies in muscle attachment areas, force magnitudes and directions of pull (Davis et al., 2010; Greaves, 2012). The advantage of FEA is that muscle forces can be distributed across the whole attachment site rather than being 
156 modelled as originating from a single centroid point, and muscle force vectors can act in three

157 dimensions rather than two.

158

159

160

MATERIALS AND METHODS

ande model creation

161

The cranium and mandible of an adult specimen of Pedetes capensis, the South African

162

springhare, were obtained from the University Museum of Zoology, Cambridge (catalogue number E.1446). The sex of the specimen was unknown, but sexual dimorphism has not been reported in this species (Offermans \& De Vree, 1989; López-Antoñanzas, 2016). The specimen was microCT scanned on the X-Tek Metris system in the Medical and Biological Engineering group, University of Hull. Voxels were isometric with dimensions of $0.052 \mathrm{~mm}$ and $0.041 \mathrm{~mm}$ for the cranium and mandible respectively.

A virtual reconstruction of the cranium was created from the scan using Avizo 8 (FEI, Hillsboro, OR). Bone and teeth were segmented as separate materials, but no differentiation was made between cortical and trabecular bone, nor between different materials within the teeth. These simplifications of the model geometry were felt to be justified as several previous studies have indicated that, whilst absolute strain magnitudes are impacted by the presence or absence of trabecular bone and different tooth materials, the large-scale patterns of deformation are relatively insensitive to such changes (Fitton et al., 2015; Toro-Ibacache et al., 2016). In order to reduce solution times and allow effective manipulation of the model in the FE software, the reconstruction was down-sampled to a voxel size of $0.21 \times 0.21 \times 0.21 \mathrm{~mm}$. The cranial reconstruction was then converted into a mesh of 2,310,268 eight-noded, cubic (first-order) elements via direct voxel conversion, implemented in VOX-FE, custom-built open-source FE software (Liu et al., 2012). The Avizo reconstruction and VOX-FE model are both available for download at https://figshare.com/articles/Springhare FEA/5082598.

\section{Material properties, constraints and loads}

Material properties were assigned to the model based on previous nano-indentation work on rodent skulls (Cox et al., 2012). Both bone and teeth were assumed to be linearly elastic isotropic with Young's moduli of 17 and $30 \mathrm{GPa}$ respectively and a Poisson's ratio of 0.3 for both. The 
187 model was constrained at both temporo-mandibular joints as well as the biting tooth. The jaw

188 joints were constrained in all three dimensions, whilst the bite points were only constrained in 189 the bite direction (i.e. orthogonal to the occlusal plane). This configuration of constraints is 190 somewhat conservative (it restricts the jaw to simple hinge movements), but has been used by a 191 number of other authors previously (Porro et al., 2013; Cuff, Bright \& Rayfield, 2015; Cox, 192 Rinderknecht \& Blanco, 2015) and provides robust conclusions with regard to mechanical 193 advantage. The number of nodes constrained at each location varied between 158 and 332 .

194

195

196

197

198

199

200

201

202

203

204

205

206

207

208

209

210

211

212

213

214

215

216

217

Loads were applied to both sides of the model to simulate the following jaw-closing muscles (see Figure 1) based on previous published data (Offermans \& De Vree, 1989, 1993): masseter (combining the superficial and deep layers), posterior masseter, ZM, IOZM, temporalis, medial pterygoid and lateral pterygoid. Unfortunately, the superficial and deep masseters could not be modelled separately, because they were recorded as a single entity in Offermans \& De Vree (1993). Muscle attachment sites were determined based on the detailed descriptions and figures in Offermans \& De Vree (1989). Muscle directions of pull (assuming a gape angle of $0^{\circ}$, i.e. teeth in occlusion) were assigned using landmarks recorded from the insertion areas on a reconstruction of the springhare mandible, created from the previously gathered microCT scans. Muscle forces were calculated by multiplying the physiological cross-sectional areas (PCSA) given in Offermans \& De Vree (1993) by an intrinsic muscle stress value of $0.3 \mathrm{Nmm}^{-2}$ (van Spronsen et al., 1989; Sharp, 2015; Tseng \& Flynn, 2015). These muscle forces were then modified based on the maximum percentage activations recorded by electromyography during incision and mastication of groundnuts (Offermans \& DeVree, 1993). Thus the relative proportions of total muscle force provided by each muscle were different in incisor biting to premolar/molar biting. Applied muscle forces for incision and mastication are given in Table 1. In order to ascertain the function of the masticatory muscles, versions of the model were created without each of the muscles in turn. The loaded FE model is shown in Figure 1.

\section{Model solution and analysis}

The model was solved for biting at each tooth along the dental arcade. Based on experimental work by Offermans \& De Vree (1990), all bites were modelled as bilateral i.e. the muscles on both sides of the skull were active with identical forces and the same tooth was loaded on each 
218 side of the dental row. Reaction forces at the biting tooth and at the jaw joints were calculated for

219 each loadcase. Bite forces were divided by the effective muscle force (equal to the sum of the

220 bite force and joint reaction forces) to calculate the mechanical advantage of the masticatory

221 system at each tooth. As a ratio, the mechanical advantage provides a useful metric for

222 comparing loadcases with different input muscle forces. It should be noted that it is a different 223 measure to the mechanical efficiency of biting used in other studies (Dumont et al., 2011; Cox et

224 al., 2012; Cox, Kirkham \& Herrel, 2013), which divides the bite force by the total adductor 225 muscle force, but does not take into account the orientation of muscle vectors. The distribution of 226 maximum $\left(\varepsilon_{1}\right.$ : predominantly tensile) and minimum $\left(\varepsilon_{3}\right.$ : predominantly compressive) principal 227 strains across the skull were examined using contour maps. Geometric morphometric methods 228 were used to analyse deformation patterns across the skull (Cox et al., 2011; Cox, Kirkham \& 229 Herrel, 2013; O’Higgins et al., 2011; McIntosh \& Cox, 2016). A set of 46 3D landmark co230 ordinates (described in Figure 2 and Table S1), based on that used in Cox, Kirkham \& Herrel 231 (2013), was recorded from each solved model as well as from the original unloaded model. As 232 changes in size are of equal significance to changes in shape during mechanical loading, the 233 landmark sets were subjected to a Procrustes size and shape analysis (O’Higgins \& Milne, 2013), 234 not a Procrustes form analysis, which gives a lower weighting to size (Fitton et al., 2015). This 235 was followed by a principal component analysis (PCA). All analyses were implemented in the 236 EVAN toolbox software (www.evan-society.org).

\section{RESULTS}

239 The absolute bite forces and joint reaction forces predicted by the model during biting at each tooth in $P$. capensis are given in Table 2. In addition, the mechanical advantage of the jaws at each bite has been calculated. It can be seen that joint reaction forces are negative and mechanical advantage exceeds one at all three molar teeth. In addition, the mechanical advantage is almost one (0.99) and the joint reaction force is close to zero $(2.8 \mathrm{~N})$ at the premolar.

The effect of removing each of the masticatory muscles on the overall mechanical advantage is given in Table 2 and shown in Figure 3. Removal of either the IOZM or the masseter causes a decrease in mechanical advantage during both incision and mastication, with removal of the 
249 increase in mechanical advantage across all cheek teeth, but little effect is seen during incisor

250 biting. Removal of the ZM causes a substantial drop in bite force at all teeth, but has little effect

251 on the mechanical advantage of the system, except at the incisor where mechanical advantage

252 increases in the absence of the ZM. Removal of the posterior masseter, temporalis or lateral

253 pterygoid results in very little change in either bite force or mechanical advantage at any of the

254 teeth, and hence the results of the models lacking these muscles have not been illustrated in

255 Figure 3 (although the numerical data is still available in Table 2).

256

257 The contour maps of principal strain distribution across the cranium of $P$. capensis during biting 258 on the incisor and first molar are shown in Figure 4. It can be seen that the highest maximum and 259 minimum principal strains are concentrated in similar areas of the skull - along the zygomatic 260 arch and up its wide ascending ramus, and across the orbital wall, especially the anterior part. 261 However, there are some differences between the strain distributions. The ascending ramus of 262 the zygomatic arch is subject to greater $\varepsilon_{1}$ strains than $\varepsilon_{3}$ strains, and thus is predominantly under 263 tension, whereas the orbital wall seems to be experiencing greater $\varepsilon_{3}$ strains and is likely mostly 264 in compression. Strains are generally greater during molar biting than incision, and there is an 265 overall caudal shift of the most highly strained regions away from the rostrum towards the orbit 266 as the bite point moves posteriorly along the tooth row.

267

Figure 4 also shows the effect of removing three of the masticatory muscles (IOZM, masseter and $\mathrm{ZM}$ ) on principal strain distributions. Despite being relatively large muscles, the impact of removing the IOZM or the masseter appears to be minimal. There are very few differences between models with all masticatory muscles applied and those without the IOZM, except for a

272 slight reduction in strain on the rostrum and in the posterior part of the orbit during incisor and molar biting. Removal of the masseter has little effect on the strains generated by incisor biting, but reduces strains across the zygomatic arch and in the anterior part of the orbit during molar biting. Elimination of the ZM from the model, however, leads to a substantial reduction in $\varepsilon_{1}$ and $\varepsilon_{3}$ strains across the skull during bites at all teeth.

The geometric morphometric analysis highlights differences in the magnitude and mode of 279 deformation between the different loadcases solved in this study. Figure 5 shows the scatter plot 
280 of the first two principal components. The first principal component encompasses $90 \%$ of the 281 variation, and the second principal component $9 \%$ of the variation. It should be noted that to be 282 able to visualise change across PC2, the axes have not been shown to the same scale. As 283 demonstrated by the warped reconstructions in Figure 5, the shape change along PC1 is mainly 284 bending of the zygomatic arch, and this axis mostly separates loaded models from the unloaded skull, incisor bites from bites on other teeth, and models with different muscles excluded from one another. In general, incisor bites result in smaller deformations than cheek tooth bites (that is, the incisor bites are found closer to the unloaded model on PC1), whereas premolar and molar bites produce very similar deformations. Models lacking the IOZM, temporalis, medial pterygoid or lateral pterygoid deform in a very similar manner to the models with all masticatory muscles, whereas removal of the posterior masseter reduces the magnitude of deformation very slightly. Removal of the masseter causes a greater reduction in cranial deformation and elimination of the ZM (the largest masticatory muscle) causes the largest reduction in deformation. Shape change along PC2 represents dorso-ventral bending of the skull and separates the four different bites along the cheek tooth row.

\section{DISCUSSION}

The results of this study support both of the hypotheses proposed here. The skull of Pedetes capensis operates as a second-class lever during biting along almost all of the cheek teeth (first hypothesis), and this effect can be largely ascribed to the presence of the IOZM muscle (second hypothesis), although the masseter is important in this regard as well.

301

302

Second-class vs third-class lever

303

The FE model of $P$. capensis indicates that the mechanical advantage of the masticatory system is greater than one and the reaction forces at the temporo-mandibular joints are negative during bites on all three molars. Furthermore, the mechanical advantage is almost one and the joint reaction force is very close to zero during premolar biting. Thus, as the bite point moves distally along the tooth row, the system switches from a third-class to a second-class lever somewhere between the premolar and first molar. In an analysis of the mandibles of two murid species, Apodemus speciosus and Cleithrionomys rufocanus, such an effect was calculated to occur 310 between the first and second molars (Satoh, 1999). The more anterior position of the effective 
311 muscle force in the springhare may be driven in large part by its unusual cranial morphology. In

312 most hystricomorph rodents, the anterior root of the zygomatic arch arises from the skull 313 approximately at the level of the first cheek tooth, but in P. capensis it is much further forward, 314 attaching to the shortened rostrum just posterior to the upper incisor (Offermans \& De Vree, 315 1989). Thus, the masticatory musculature, as a whole, is more rostrally positioned than in most 316 other rodents, and so the jaw becomes a second-class lever at more anterior position along the 317 tooth row.

319 The prediction of large tensile forces at the jaw joints of the springhare is a result that is at odds with some published work on mammalian masticatory biomechanics. It has been argued that the capsule and ligaments of the mammalian jaw joint are poorly suited to resisting high tensile forces (Greaves, 2000, 2012), and that the mammalian masticatory system has evolved to maintain the resultant muscle force within the posterior third of the jaw ramus in order to prevent instability and dislocation of the jaws (Greaves, 1978, 1982, 2000; Spencer, 1998, 1999). The mismatch between the results presented here and this earlier work may be the product of insufficient knowledge of muscle recruitment in springhare mastication and also the limitations of static FE models. Although the muscle forces were based on in vivo work that measured the degree to which each muscle was activated during biting (Offermans \& De Vree, 1993), a single set of values was used for all molar bites; the only variation in muscle recruitment was between incisor and cheek teeth bites. It has been shown that muscle recruitment can vary a great deal from tooth to tooth, and even between bites on the same tooth (Cleuren, Aerts \& De Vree, 1995). Furthermore, the percentage activations used to calculate muscle force are the maximum applied at any point during the masticatory cycle. Obviously, the recruitment of each muscle changes as the teeth are brought into and out of occlusion, but a static model cannot reflect this. Thus, the results here indicate that jaw is a second-class lever in molar biting, but this only holds true for the muscle recruitment pattern applied to the model. In reality, the springhare may modulate fibre recruitment within its complex set of muscles to maintain the jaws as a third-class lever even at the distal molars. For instance, the external pterygoid may increase in activation during distal molar biting to resist dislocation of the jaws as has been suggested to occur in murids (Satoh, 1999). 
342 Even after taking the limitations of the model into consideration, it is clear that the springhare

343 has the potential to generate very high bite forces at its molar teeth. Moreover, even if not quite a 344 second-class lever these bites are very efficient, so high bite forces can be produced without

345 having to massively increase the overall adductor muscle mass. It is likely that $P$. capensis has

346 evolved this highly efficient feeding system in order to cope with the demands of the arid 347 environment in which it lives (Peinke \& Brown, 2003). Springhares are herbivorous, feeding 348 almost exclusively on grasses (Peinke \& Brown, 2006). Although they are known to eat the 349 leaves, springhares tend to favour underground storage organs, such as rhizomes and tubers, 350 particularly during autumn and winter when nutritional reserves are transferred away from leaves 351 and into the leaf bases and roots (Peinke \& Brown, 2006). These storage organs tend to be 352 mechanically demanding to eat, requiring a great deal of mastication to break down, which may 353 have driven the evolution of the highly efficient masticatory system of springhares. The 354 disadvantage of the masticatory arrangement seen in P. capensis is that the rostral position of 355 many of the jaw-closing muscles is likely to severely limit maximum gape. However, given their 356 preferred diet of grasses, these limitations are not likely to impact the ability of springhares to 357 feed effectively.

Function of the masticatory muscles

360 The virtual ablation experiments, in which masticatory muscles were sequentially removed from 361 the FE model, show that the IOZM is the most important muscle in converting the masticatory 362 system from a third-class to second-class lever in molar biting, as predicted by the second 363 hypothesis. When the IOZM is removed, the mechanical advantage decreases, indicating that 364 more force is being directed towards the jaw joints. This has the effect that the point at which the 365 system switched from operating as a third-class to a second-class lever moves back to 366 somewhere between the first and second molars. Thus, this result supports the idea proposed by 367 Becht (1953) that the function of the IOZM is to convert the masticatory system to a second368 class lever during molar biting, at least in P. capensis. Removal of the IOZM had very little 369 impact on the distribution and magnitudes of strain across the skull (Figure 4), nor did it greatly 370 change the overall deformation of the skull during biting (Figure 5), as has also been noted in 371 another species of rodent, Laonastes aenigmamus (Cox, Kirkham \& Herrel, 2013). Thus, it 372 appears that the increase in mechanical advantage gained by the presence of an IOZM muscle 
373 does not come at the cost of greatly increased strain or deformation, either in the region of the

374 IOZM origin or elsewhere on the skull. In addition, the development of the IOZM in P. capensis

375 may be a response to the need to generate large forces at the incisors, such as during the cracking

376 of nutshells (Offermans \& De Vree, 1990) or gnawing of roots and tubers (Peinke \& Brown,

377 2006). The anterior position of the IOZM on the skull means that improvements in incisor bite

378 force can be achieved without an excessive increase in muscle size.

379

380 The ZM is one of the largest masticatory muscles in the springhare (Offermans \& De Vree, 381 1993), which is unusual among rodents; usually the ZM is smaller than the superficial and deep

382

383

384

385

386

387

388

389

390

391

392

393

394

395

396

397

398

399

400

401

402

403 masseters and the IOZM (Turnbull, 1970; Woods, 1972; Cox \& Jeffery, 2011; Baverstock, Jeffery \& Cobb, 2013; Becerra et al., 2014). Despite its large size, the removal of the ZM from the FE model had very little effect on the efficiency of the masticatory system i.e. the mechanical advantage and joint reaction force remained largely the same. Thus, by virtue of being large, the $\mathrm{ZM}$ is an important muscle for increasing overall bite force, but its presence does not alter the efficiency of the system a great deal. However, the ZM does have a large effect on the deformation of the springhare skull during biting. The GMM analysis showed that elimination of the ZM greatly reduces the magnitude of deformation experienced by the skull (Figure 5), much more so than any other masticatory muscle. This appears to be a consequence of the attachment site of the ZM on the zygomatic arch. As has been found in other FEA studies of mammal skulls (Bright, 2012; Cox et al., 2012; Fitton et al., 2012), the morphology of the zygomatic arch makes it susceptible to larger deformations than other parts of the skull. Indeed, in this study, deformations of the zygoma overwhelm deformations in all other parts of the skull, as can be seen from the warped reconstructions in Figure 5. The large size and location of the $\mathrm{ZM}$ in $P$. capensis leads to it being the principal generator of zygomatic strain and deformation. This can be seen in Figure 4, where removal of the ZM vastly reduces strain in the zygomatic arch.

It has been suggested that the large zygomatic strains seen in many FEA studies of mammalian skulls may be artificial and the result of a failure to incorporate important soft tissue structures into the models. In particular, the temporal fascia has been shown to resist inferior bending of the zygomatic arch in an FE model of a macaque (Curtis et al., 2011). This is unlikely to be the case here as no temporal fascia was reported by Offermans \& De Vree (1989) in their dissection of a 
404 springhare. Furthermore, the temporalis is extremely small in P. capensis, and the temporal 405 region is positioned distinctly caudal the zygomatic arch, which would reduce the ability of a 406 temporal fascia to counteract ventral deflection of the zygomatic arch. However, there is an 407 aponeurosis attached extensively around the margin of the infraorbital fossa (Offermans \& De 408 Vree, 1989), which may have the potential to resist some bending in the anterior part of the 409 zygomatic arc and its ascending ramus. Further work, both ex vivo dissection and in silico modelling, is necessary to understand the biomechanical consequence of this aponeurosis.

412 The masseter has been shown to have a similar effect to the IOZM with regard to bite force, 413 although not quite to the same extent. It, too, appears to shift the resultant masticatory muscle 414 force anteriorly along the rostrum, thus directing force towards the biting tooth and away from 415 the jaw joints. Removal of the masseter has much the same effect as removing the IOZM - the mechanical advantage is decreased and the point at which the system becomes a second-class

417 lever is shifted posteriorly along the tooth row. Unfortunately for this study, Offermans \& 418 DeVree (1993) did not separate the superficial and deep masseter when measuring PCSA, so the two muscles could not be modelled separately in the FEA. However, the illustrations in Offermans \& DeVree (1989) indicate that the fibres of the superficial masseter have a more horizontal alignment than those of the deep masseter (as in most rodents, e.g. Turnbull, 1970), so it is likely that the superficial masseter is the more important division of the masseter with regard to the operation of the jaw as a second-class lever. In terms of cranial deformations, the masseter

424 has a similar, but lesser, effect to the ZM. Owing to its attachment to the zygomatic arch, the action of the masseter generates inferior bending of the arch, and thus its removal tends to reduce global deformation of the springhare cranium (Figure 5). It can also be seen that that removal of

427 the masseter causes a slight reduction in zygomatic and orbital strains during molar biting 428 (Figure 4).

The medial pterygoid, because of its posterior line of action, tends to direct muscle force away 431 from the teeth and towards the jaw joints, unlike the IOZM and masseter. Thus removal of the medial pterygoid increased the mechanical advantage of the masticatory system. Overall, the presence of the medial pterygoid increases bite force because it increases the total input adductor muscle force, but it does so in a somewhat inefficient manner. Thus, although the medial 
435 pterygoid has a relatively large PCSA, it has a relatively small effective muscle force owing to

436 its orientation. However, it has been shown that the medial pterygoid is important in other 437 aspects of masticatory biomechanics, notably as a counterbalance to the lateral pull of the 438 masseter, thereby preventing wishboning of the mandible (eversion of the lower border and 439 angular process) and reducing tensile strains at the symphysis (Hiiemae, 1971; Satoh, 1998; Cox 440 \& Jeffery, 2015).

441

442 The posterior masseter, temporalis and lateral pterygoid are very small compared to the other 443 masticatory muscles in $P$. capensis, each providing less than $11 \%$ of the total adductor muscle 444 force. Hence, the impact of their removal on bite force and mechanical advantage was minimal. 445 Similarly, removal of these muscles had a very limited impact on the overall deformation of the 446 skull (Figure 5). The models without the temporalis and lateral pterygoid can barely be 447 distinguished from the models with all masticatory muscles. The models without a posterior 448 masseter show a very slight reduction in the magnitude of cranial deformation. This is because 449 the posterior masseter attaches to the caudal part of the zygomatic arch and thus is able to cause a 450 small amount of posterior deflection. It is likely that these muscles contribute to aspects of the 451 masticatory process other than bite force generation, especially the antero-posterior movements 452 of the mandible relative to the skull that are common to rodents. The temporalis, whilst clearly 453 too small to be a powerful elevator of the jaw as in myomorphs (Hiiemae, 1971; Gorniak, 1977), 454 may have a braking role during the power stroke of chewing (Byrd, 1981), and the lateral 455 pterygoid may be important in protraction of the mandible (Weijs \& Dantuma, 1975; Gorniak, 1977) or in resisting tensile forces at the temporo-mandibular joint as mentioned above (Satoh, 457 1999).

\section{CONCLUSIONS}

460 The masticatory system of $P$. capensis has been shown to have the potential to act as a second461 class lever along the majority of the cheek tooth row and, as predicted by Becht (1953), the IOZM is a particularly important muscle in the switch from third-class to second-class lever mechanics. It should be noted that masseter also plays an important role in this regard. This analysis of muscle function is, of course, specific to $P$. capensis and further analyses of other species are necessary to determine whether the conclusions hold true for other rodents. However, 
466 the position of the IOZM, far forward on the rostrum, makes it likely that it will have some role

467 to play in increasing the mechanical advantage of the masticatory system in most hystricomorph 468 rodents (the exact scale of the effect being dependent on the size of the IOZM relative to the 469 other masticatory muscles). Previous research has suggested that, amongst rodents, sciuromorphs 470 are adapted for efficient gnawing at the incisors, whereas hystricomorphs are adapted to efficient 471 grinding at the molars (Cox et al., 2012). Druzinsky (2010) determined that of all the masticatory 472 muscles, it is the anterior deep masseter that confers efficacious incisor bites in sciuromorphs. 473 Here, it is indicated that the IOZM provides efficiency in molar bites in hystricomorphs, without 474 substantially increasing strains across the skull or the magnitude of cranial deformation. This 475 may go some way to explaining why hystricomorphy has evolved convergently at least four 476 times within the rodents.

477

\section{ACKNOWLEDGEMENTS}

479 The author thanks Matt Lowe and Rob Asher (University Museum of Zoology, Cambridge) for 480 the loan of the specimen, and Sue Taft and Michael Fagan (Medical and Biological Engineering 481 Group, University of Hull) for microCT scanning. Thanks are due to Paul O'Higgins for help 482 with geometric morphometrics, and to three anonymous reviewers for their useful comments that 483 helped improve this manuscript.

484

\section{REFERENCES}

Baverstock H, Jeffery NS, Cobb SN. 2013. The morphology of the mouse masticatory musculature. Journal of Anatomy 223: 46-60.

Becht G. 1953. Comparative biologic-anatomical researcher on mastication in some mammals. Proceedings of the Koninklijke Nederlandse Akademie van Wetenschappen. Series C 56: 508-526.

Becerra F, Casinos A, Vassallo AI. 2013. Biting performance and skull biomechanics of a chisel-tooth digging rodent (Ctenomys tuconax; Caviomorpha; Octodontoidea). Journal of Experimental Zoology 319A: 74-85.

Becerra F, Echeverría AI, Casinos A, Vassallo AI. 2014. Another one bites the dust: bite force and ecology in three caviomorph rodents (Rodentia, Hystricognathi). Journal of Experimental Zoology 283A: 220-232. 
497

498

499

500

501

502

503

504

505

506

507

508

509

510

511

512

513

514

515

516

517

518

519

520

521

522

523

524

525

Bramble DM. 1978. Origin of the mammalian fedding complex: Models and mechanisms. Paleobiology 4: 271-301.

Bright JA. 2012. The importance of craniofacial sutures in biomechanical finite element models of the domestic pig. PLOS ONE 7: e31769.

Byrd KE. 1981. Mandibular movement and muscle activity during mastication in the guinea pig (Cavia porcellus). Journal of Morphology 170: 147-169.

Cleuren J, Aerts P, De Vree F. 1995. Bite and joint force analysis in Caiman crocodilus. Belgian Journal of Zoology 125, 79-94.

Cornette R, Herrel A, Cosson, J-F, Poitevin F, Baylac M. 2012. Rapid morpho-functional changes among insular populations of the great white-toothed shrew. Biological Journal of the Linnean Society 107: 322-331.

Cox PG, Fagan MJ, Rayfield EJ, Jeffery N. 2011. Finite element modelling of squirrel, guinea pig and rat skulls: using geometric morphometrics to assess sensitivity. Journal of Anatomy 219: 696-709.

Cox PG, Jeffery N. 2011. Reviewing the jaw-closing musculature in squirrels, rats and guinea pigs with contrast-enhanced microCT. Anatomical Record 294: 915-928.

Cox PG, Jeffery N. 2015. The muscles of mastication and the function of the medial pterygoid. In: Cox PG, Hautier L, eds. Evolution of the Rodents: Advances in Phylogeny, Functional Morphology and Development. Cambridge: Cambridge University Press, 350-372.

Cox PG, Kirkham J, Herrel A. 2013. Masticatory biomechanics of the Laotian rock rat, Laonastes aenigmamus, and the function of the zygomaticomandibularis muscle. PeerJ 1: e160.

Cox PG, Rayfield EJ, Fagan MJ, Herrel A, Pataky TC, Jeffery N. 2012. Functional evolution of the feeding system in rodents. PLoS ONE 7: e36299.

Cox PG, Rinderknecht A, Blanco RE. 2015. Predicting bite force and cranial biomechanics in the largest fossil rodent using finite element analysis. Journal of Anatomy 226: 215-223.

Crompton AW. 1963. The evolution of the mammalian jaw. Evolution 17: 431-439.

Cuff, AR, Bright JA, Rayfield EJ. 2015. Validation experiments on finite element models of an ostrich (Struthio camelus) cranium. PeerJ 3: e1294. 
526 Curtis N, Witzel U, Fitton LC, O'Higgins P, Fagan MJ. 2011. The mechanical significance of 527 the temporal fasciae in Macaca fascicularis: an investigation using finite element $528 \quad$ analysis. Anatomical Record 294: 1178-1190.

529 Davis DD. 1955. Masticatory apparatus in the spectacled bear Tremarctos ornatus. Fieldiana $530 \quad$ (Zoology) 37: 25-46.

531 Davis JL, Santana SE, Dumont ER, Grosse IR. 2010. Predicting bite force in mammals: two532 dimensional versus three-dimensional lever models. Journal of Experimental Biology 213: 1844-1851.

Druzinsky RE. 2010. Functional anatomy of incisal biting in Aplondontia rufa and sciuromorph rodents - Part 2: Sciuromorphy is efficacious for production of force at the incisors. Cells Tissues Organs 192: 50-63.

Dumont ER, Davis JL, Grosse IR, Burrows AM. 2011. Finite element analysis of performance in the skulls of marmosets and tamarins. Journal of Anatomy 218: 151-162.

Fabre P-H, Hautier L, Dimitrov D, Douzery EJP. 2012. A glimpse on the pattern of rodent diversification: a phylogenetic approach. BMC Evolutionary Biology 12: 88.

Figueirido B, Tseng JZ, Serrano-Alarcón FJ, Martín-Serra A, Pastor JF. 2014. Threedimensional computer simulations of feeding behaviour in red and giant pandas relate skull biomechanics with dietary niche partitioning. Biology Letters 10: 20140196.

Fitton LC, Shi JF, Fagan MJ, O'Higgins P. 2012. Masticatory loadings and cranial deformation in Macaca fascicularis: a finite element analysis sensitivity study. Journal of Anatomy 221: 55-68.

Fitton LC, Proa M, Rowland C, Toro-Ibacache V, O'Higgins P. 2015. The impact of simplifications on the performance of a finite element model of a Macaca fascicularis cranium. Anatomical Record 298: 107-121.

Gingerich PD. 1979. The human mandible: Lever, link or both? American Journal of Physical Anthropology 51: 135-138.

Gorniak GC. 1977. Feeding in golden hamsters, Mesocricetus auratus. Journal of Morphology 154: 427-458.

Greaves WS, 1978. The jaw lever system in ungulates: a new model. Journal of Zoology 184: 271-285. 
556 Greaves WS. 1982. A mechanical limitation on the position of the jaw muscles of mammals: the

557

558

559

560

561

562

563

564

565

566

567

568

569

570

571

572

573

574

575

576

577

578

579

580

581

582

583

584

585

586 one-third rule. Journal of Mammalogy 63: 261-266.

Greaves WS. 2000. Location of the vector of jaw muscle force in mammals. Journal of Morphology 243: 293-299.

Greaves WS. 2012. The Mammalian Jaw: A Mechanical Analysis. Cambridge: Cambridge University Press.

Hautier L, Cox PG, Lebrun R. 2015. Grades and clades among rodents: the promise of geometric morphometrics. In: Cox PG, Hautier L, eds. Evolution of the Rodents: Advances in Phylogeny, Functional Morphology and Development. Cambridge: Cambridge University Press, 277-299.

Hiiemae K. 1971. The structure and function of the jaw muscles in the rat (Rattus norvegicus L.) III. The mechanics of the muscles. Zoological Journal of the Linnean Society 50: 111132.

Kerr A. 2010. Introductory Biomechanics. Edinburgh: Churchill Livingstone.

Kupczik K, Dobson CA, Fagan MJ, Crompton RH, Oxnard CE, O'Higgins P. 2007. Assessing mechanical function of the zygomatic region in macaques: validation and sensitivity testing of finite element models. Journal of Anatomy 210: 41-53.

Liu J, Shi L, Fitton LC, Phillips R, O’Higgins P, Fagan MJ. 2012. The application of muscle wrapping to voxel-based finite element models of skeletal structures. Biomechanics and Modeling in Mechanobiology 11: 35-47.

López-Antoñanzas R. 2016. Family Pedetidae (Springhares). In: Wilson DE, Lacher TE, Mittermeier RA, eds. Handbook of the Mammals of the World. Volume 6: Lagomorphs and Rodents I. Barcelona: Lynx Edicions, 280-287.

Mansour RM, Reynik RJ. 1975. In vivo occlusal forces and moments: I. Forces measured in terminal hinge position and associated moments. Journal of Dental Research 54: 114120.

McCabe K, Henderson K, Pantinople J, Richards HL, Milne N. 2017. Curvature reduces bending strains in the quokka femur. PeerJ 5: e3100.

McIntosh AF, Cox PG. 2016. The impact of gape on the performance of the skull in chiseltooth digging and scratch digging mole-rats (Rodentia: Bathyergidae). Royal Society Open Science 3: 160568. 
587 Offermans M, De Vree F. 1989. Morphology of the masticatory apparatus in the springhare, 588 Pedetes capensis. Journal of Mammalogy 70: 701-711.

589 Offermans M, De Vree F. 1990. Mastication in springhares, Pedetes capensis: A $590 \quad$ cineradiographic study. Journal of Morphology 205 : 353-367.

591 Offermans M, De Vree F. 1993. Electromyography and mechanics of mastication in the 592 springhare Pedetes capensis (Rodentia, Pedetidae). Belgian Journal of Zoology 123: 231$593 \quad 261$.

594 O'Hare LMS, Cox PG, Jeffery N, Singer ER. 2013. Finite element analysis of stress in the 595 equine proximal phalanx. Equine Veterinary Journal 45: 273-277.

596 O’Higgins P, Cobb SN, Fitton LC, Gröning F, Phillips R, Liu J, Fagan MJ. 2011. 597 Combining geometric morphometrics and functional simulation: an emerging toolkit for $598 \quad$ virtual functional analyses. Journal of Anatomy 218: 3-15.

599 O'Higgins P, Milne N. 2013. Applying geometric morphometrics to compare changes in size 600 and shape arising from finite elements analysis. Hystrix 24: 126-132.

601 Peinke DM, Brown CR. 2003. Metabolism and thermoregulation in the springhare (Pedetes 602 capensis). Journal of Comparative Physiology B 173: 347-353.

603 Peinke DM, Brown CR. 2006. Habitat use by the southern springhare (Pedetes capensis) in the 604 Eastern Cape Province, South Africa. South African Journal of Wildlife Research 36: 605 103-111.

Porro LB, Metzger KA, Iriarte-Diaz J, Ross CF. 2013. In vivo bone strain and finite element modelling of the mandible of Alligator mississippiensis. Journal of Anatomy 223: 195227.

Rayfield EJ. 2007. Finite element analysis and understanding the biomechanics and evolution of living and fossil organisms. Annual Review of Earth and Planetary Science 35: 541-576.

Richmond BG, Wright BW, Grosse I, Dechow PC, Ross CF, Spencer MA, Strait DS. 2005. Finite element analysis in functional morphology. Anatomical Record Part A 283A: 259274.

Ross CF, Berthaume MA, Dechow PC, Iriarte-Diaz J, Porro LB, Richmond BG, Spencer MA, Strait D. 2011. In vivo bone strain and finite-element modeling of the craniofacial haft in catarrhine primates. Journal of Anatomy 218: 112-141. 
617 Santana SE. 2015. Quantifying the effect of gape and morphology on bite force: biomechanical

618

619

620

621

622

623

624

625

626

627

628

629

630

631

632

633

634

635

636

637

638

639

640

641

642

643

644

645

646 modelling and in vivo measurements in bats. Functional ecology 30: 557-565.

Satoh K. 1998. Balancing function of the masticatory muscles during incisal biting in two murid rodents, Apodemus speciosus and Clethrionomys rufocanus. Journal of Morphology 236: 49-56.

Satoh K. 1999. Mechanical advantage of area of origin for the external pterygoid muscle in two murid rodents, Apodemus speciosus and Clethrionomys rufocanus. Journal of Morphology 240: 1-14.

Satoh K, Iwaku F. 2006. Jaw muscle functional anatomy in northern grasshopper mouse, Onychomys leucogaster, a carnivorous murid. Journal of Morphology 267: 987-999.

Satoh K, Iwaku F. 2009. Structure and direction of jaw adductor muscles as herbivorous adaptations in Neotoma Mexicana (Muridae, Rodentia). Zoomorphology 128: 339-348.

Sharp A. 2015. Comparative finite element analysis of the cranial performance of four herbivorous marsupials. Journal of Morphology 276: 1230-1243.

Spencer MA. 1998. Force production in the primate masticatory system: electromrographic test of biomechanical hypotheses. Journal of Human Evolution 34: 25-54.

Spencer MA. 1999. Constraints on masticatory system evolution in anthropoid primates. American Journal of Physical Anthropology 108: 483-506.

Thomason JJ, 1991. Cranial strength in relation to estimated biting forces in some mammals. Canadian Journal of Zoology 69: 2326-2333.

Toro-Ibacache V, Fitton LC, Fagan MJ, O'Higgins P. 2016. Validity and sensitivity of a human cranial finite element model: implications for comparative studies of biting performance. Journal of Anatomy 228: 70-84.

Tseng ZJ, Flynn JJ. 2015. Are cranial biomechanical simulation data linked to known diets in extant taxa? A method for applying diet-biomechanics linkage models to infer feeding capability of extinct species. PLoS ONE 10: e0124020.

Tsouknidas A, Jimenez-Rojo L, Karatsis E, Michailidas N, Mitsiadis TA. 2017. A biorealistic finite element model to evaluate the effect of masticatory loadings on mouse mandible-related tissues. Frontiers in Physiology 8: 273.

Turnbull WD. 1970. Mammalian masticatory apparatus. Fieldiana (Geology) 18: 147-356. 
647

648

649

650

651

652

653

654

655

656

657

658

659

660

661

662

663

664

665

666

667

668

669

670

671

672

673

674

675

676

677

van Spronsen PH, Weijs WA, Valk J, Prahl-Andersen B, van Ginkel FC. 1989. Comparison of jaw-muscle bite-force cross-sections obtained by means of magnetic resonance imaging and high-resolution CT scanning. Journal of Dental Research 68: 1765-1770.

Weijs WA, Dantuma R. 1975. Electromyography and mechanics of mastication in the albino rat. Journal of Morphology 146: 1-34.

Wilson DE, Reeder DM. 2005. Mammal Species of the World. Baltimore: Johns Hopkins Press.

Wood AE. 1965. Grades and clades among rodents. Evolution 19: 115-130.

Woods CA. 1972. Comparative myology of jaw, hyoid and pectoral appendicular regions of New and Old World hystricomorph rodents. Bulletin of the American Museum of Natural History 147: 115-198.

\section{FIGURE LEGENDS}

Figure 1. FE model showing muscle attachment sites and vectors. Skull of Pedetes capensis shown in (A) lateral and (B) dorso-lateral view. Key: blue, masseter; brown, posterior masseter; dark green, ZM; light green, IOZM; red, temporalis; orange, medial pterygoid; yellow, lateral pterygoid.

Figure 2. Landmarks used in GMM analysis of skull deformations. Reconstruction of skull of Pedetes capensis in (A) dorsal, (B) ventral and (C) left lateral view. Landmarks 11-28 recorded on both sides of the skull. Landmark descriptions are given in Table S1.

Figure 3. Mechanical advantage at each tooth predicted by FE model. Abbreviations: I, incisor; PM, premolar; M1, first molar; M2, second molar; M3, third molar. Data for models with posterior masseter, temporalis and lateral pterygoid removed available in Table 2 but not illustrated here.

Figure 4. Predicted principal strains across the skull of Pedetes capensis during incisor and first molar biting. A-H: maximum $\left(\varepsilon_{1}\right)$ principal strains during incisor (A-D) and M1 (EH) biting; I-P: minimum $\left(\varepsilon_{3}\right)$ principal strains during incisor (I-L) and M1 (M-P) biting. A,E,I,M: model with all masticatory muscles included; B,F,J,N: model with IOZM excluded; C,G,K,O: model with masseter excluded; D,H,L,P: model with ZM excluded. 
679 Figure 5. GM analysis of cranial deformations in Pedetes capensis. Plot of the first two 680 principal components from a GM analysis of 46 landmarks and 41 models. Axes not to scale. Cranial reconstructions indicate shape changes (x400) along axes. 


\section{Table $\mathbf{1}$ (on next page)}

Muscle forces applied to each side of the model.

PCSA and percentage activations of each muscle from Offermans \& De Vree (1993). 
1 Table 1. Muscle forces applied to each side of the model. PCSA and percentage activations of each 2 muscle from Offermans \& De Vree (1993).

3

\begin{tabular}{|c|c|c|c|c|c|c|}
\hline & \multirow{2}{*}{$\begin{array}{l}\text { PCSA } \\
\left(\mathrm{cm}^{2}\right)\end{array}$} & \multirow{2}{*}{$\begin{array}{c}\text { Maximum } \\
\text { force (N) }\end{array}$} & \multicolumn{2}{|c|}{$\%$ activation } & \multicolumn{2}{|c|}{ Applied force (N) } \\
\hline & & & Incision & Mastication & Incision & Mastication \\
\hline Masseter & 2.886 & 86.58 & 20 & 70 & 17.32 & 60.61 \\
\hline Posterior masseter & 0.654 & 19.62 & 0 & 40 & 0.00 & 7.85 \\
\hline ZM & 3.360 & 100.80 & 60 & 100 & 60.48 & 100.80 \\
\hline IOZM & 2.244 & 67.32 & 100 & 60 & 67.32 & 40.39 \\
\hline Temporalis & 0.516 & 15.48 & 0 & 30 & 0.00 & 4.64 \\
\hline Medial pterygoid & 1.130 & 33.90 & 15 & 90 & 5.09 & 30.51 \\
\hline Lateral pterygoid & 0.519 & 15.57 & 60 & 70 & 9.34 & 10.90 \\
\hline
\end{tabular}

4 


\section{Table 2 (on next page)}

Bite force, joint reaction force and mechanical advantage of each model. 
1 Table 2. Bite force, joint reaction force and mechanical advantage of each model.

2

\begin{tabular}{|l|l|l|l|l|l|l|l|l|}
\hline & $\begin{array}{l}\text { All } \\
\text { muscles }\end{array}$ & $\begin{array}{l}\text { No } \\
\text { masseter }\end{array}$ & $\begin{array}{l}\text { No } \\
\text { posterior } \\
\text { masseter }\end{array}$ & No ZM & $\begin{array}{l}\text { No } \\
\text { IOZM }\end{array}$ & $\begin{array}{l}\text { No } \\
\text { temporalis }\end{array}$ & $\begin{array}{l}\text { No } \\
\text { medial } \\
\text { pterygoid }\end{array}$ & $\begin{array}{l}\text { No } \\
\text { lateral } \\
\text { pterygoid }\end{array}$ \\
\hline Bite force & & & & & & & \\
\hline I & 154.6 & 138.3 & 154.3 & 122.5 & 49.9 & 154.3 & 152.0 & 154.3 \\
\hline PM & 347.5 & 244.4 & 345.7 & 247.4 & 232.5 & 346.6 & 319.9 & 348.7 \\
\hline M1 & 395.6 & 279.0 & 393.5 & 280.7 & 265.7 & 394.6 & 362.5 & 397.4 \\
\hline M2 & 457.7 & 323.0 & 455.2 & 324.7 & 307.8 & 456.4 & 418.8 & 459.8 \\
\hline M3 & 539.6 & 380.8 & 536.7 & 382.9 & 362.7 & 538.1 & 494.1 & 541.9 \\
\hline Joint reaction force & & & & & & & \\
\hline I & 85.9 & 83.0 & 86.2 & 39.8 & 56.0 & 86.2 & 79.3 & 154.3 \\
\hline PM & 2.8 & 29.2 & 3.4 & -2.3 & 38.8 & -0.4 & -26.9 & 9.3 \\
\hline M1 & -45.3 & -5.4 & -44.4 & -35.7 & 5.5 & -48.3 & -69.6 & -39.5 \\
\hline M2 & -107.3 & -49.4 & -106.1 & -79.7 & -36.4 & -110.2 & -125.9 & -102.0 \\
\hline M3 & -189.2 & -107.1 & -187.5 & -137.9 & -91.3 & -191.8 & -201.1 & -184.0 \\
\hline Mechanical advantage & & & & & & \\
\hline I & 0.64 & 0.62 & 0.64 & 0.75 & 0.47 & 0.64 & 0.66 & 0.50 \\
\hline PM & 0.99 & 0.89 & 0.99 & 1.01 & 0.86 & 1.00 & 1.09 & 0.97 \\
\hline M1 & 1.13 & 1.02 & 1.13 & 1.15 & 0.98 & 1.14 & 1.24 & 1.11 \\
\hline M2 & 1.31 & 1.18 & 1.30 & 1.33 & 1.13 & 1.32 & 1.43 & 1.28 \\
\hline M3 & 1.54 & 1.39 & 1.54 & 1.56 & 1.34 & 1.55 & 1.69 & 1.51 \\
\hline
\end{tabular}




\section{Figure 1}

FE model showing muscle attachment sites and vectors.

Skull of Pedetes capensis shown in (A) lateral and (B) dorso-lateral view. Key: blue, masseter; brown, posterior masseter; dark green, ZM; light green, IOZM; red, temporalis; orange, medial pterygoid; yellow, lateral pterygoid.
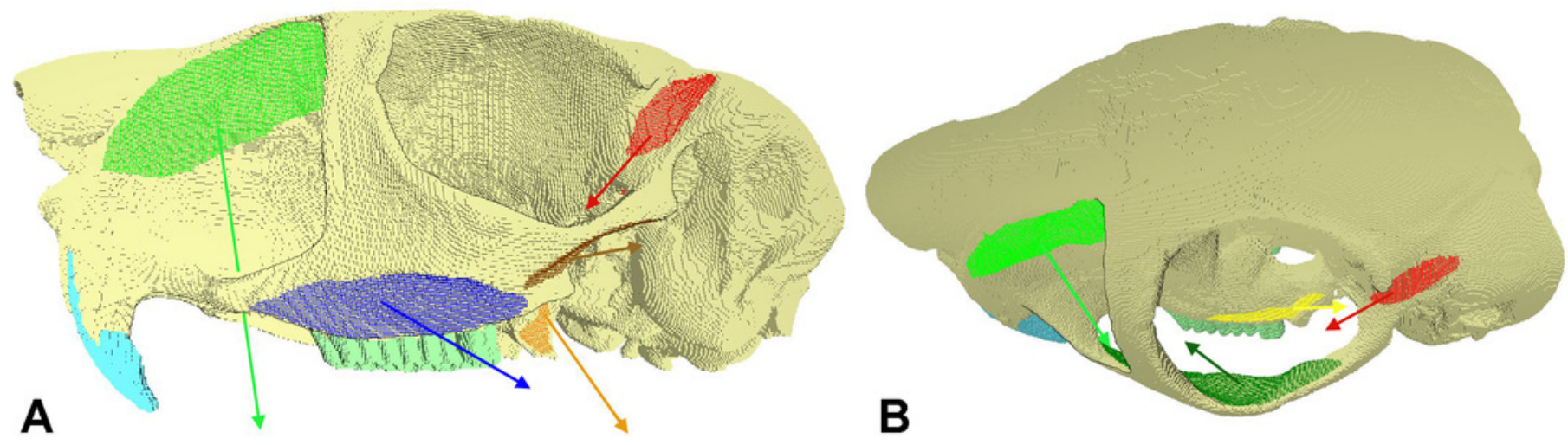
Figure 2

Landmarks used in GMM analysis of skull deformations.

Reconstruction of skull of Pedetes capensis in (A) dorsal, (B) ventral and (C) left lateral view. Landmarks 11-28 recorded on both sides of the skull. Landmark descriptions are given in Table S1. 

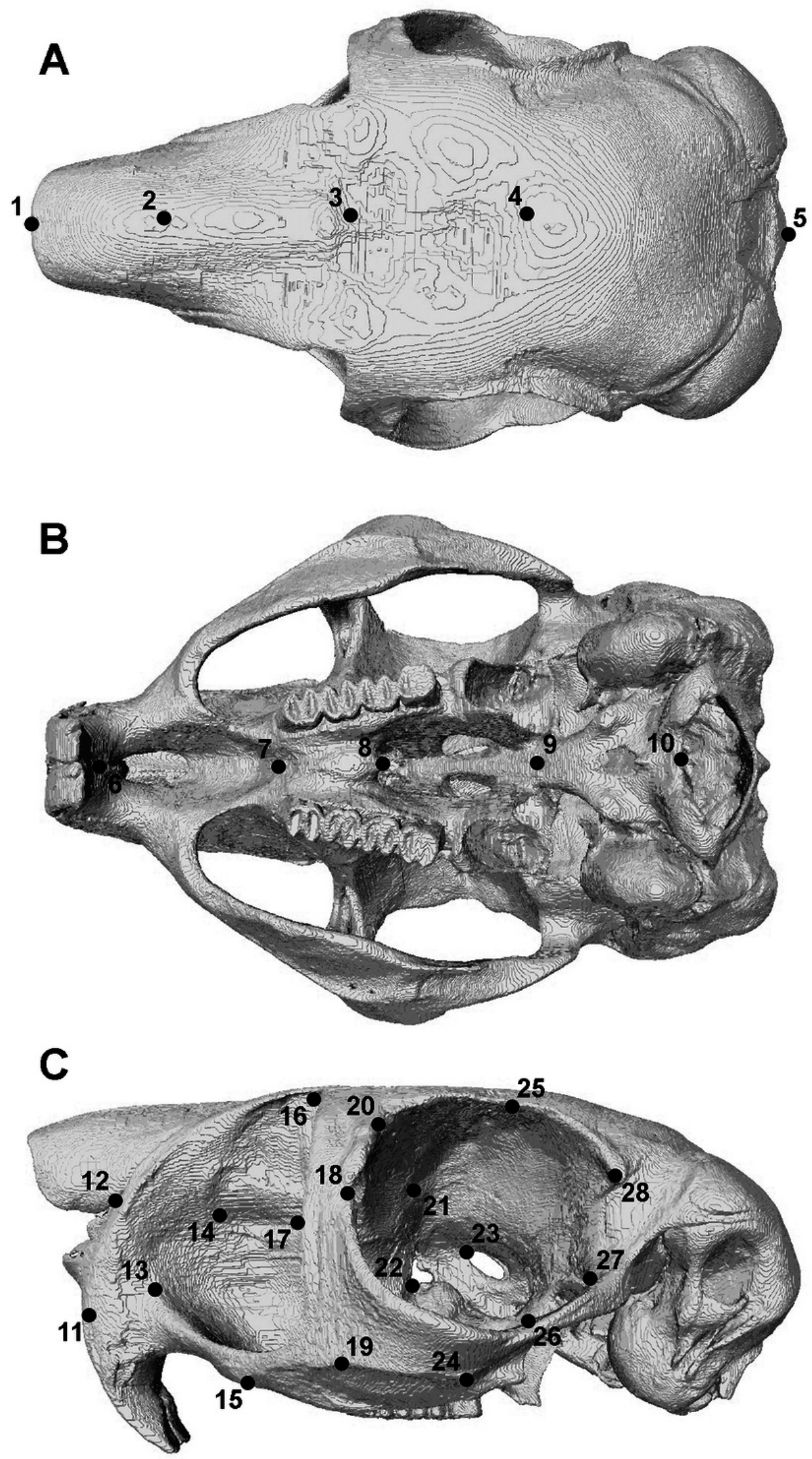
Figure 3

Mechanical advantage at each tooth predicted by FE model.

Abbreviations: I, incisor; PM, premolar; M1, first molar; M2, second molar; M3, third molar. Data for models with posterior masseter, temporalis and lateral pterygoid removed available in Table 2 but not illustrated here.

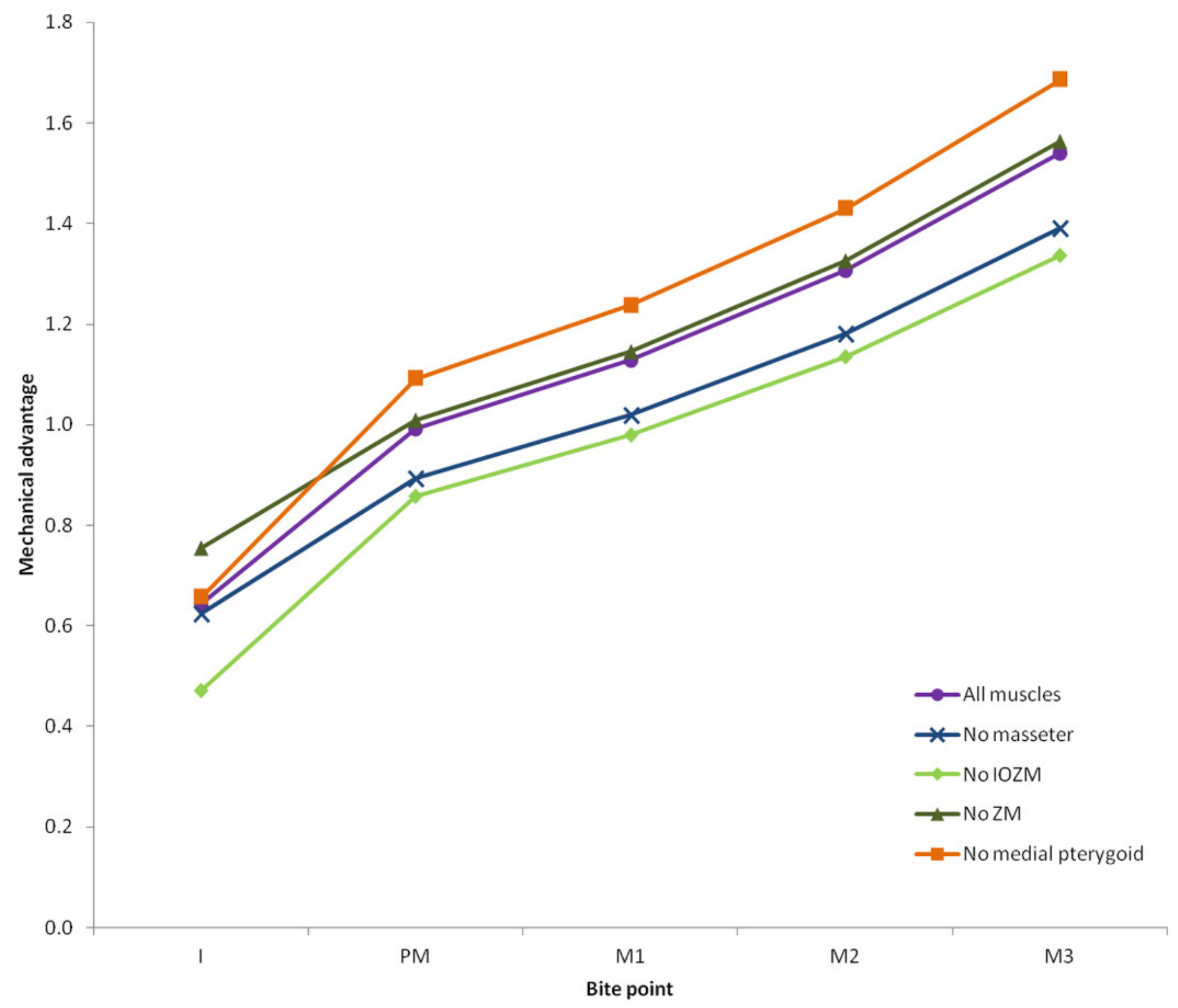




\section{Figure 4}

Predicted principal strains across the skull of Pedetes capensis during incisor and first molar biting.

A-H: maximum $\left(\varepsilon_{1}\right)$ principal strains during incisor (A-D) and M1 (E-H) biting; I-P: minimum $\left(\varepsilon_{3}\right)$ principal strains during incisor (I-L) and M1 (M-P) biting. A,E,I,M: model with all masticatory muscles included; B,F,J,N: model with IOZM excluded; C,G,K,O: model with masseter excluded; D,H,L,P: model with ZM excluded.




Figure 5

GM analysis of cranial deformations in Pedetes capensis.

Plot of the first two principal components from a GM analysis of 46 landmarks and 41 models. Axes not to scale. Cranial reconstructions indicate shape changes (x400) along axes.

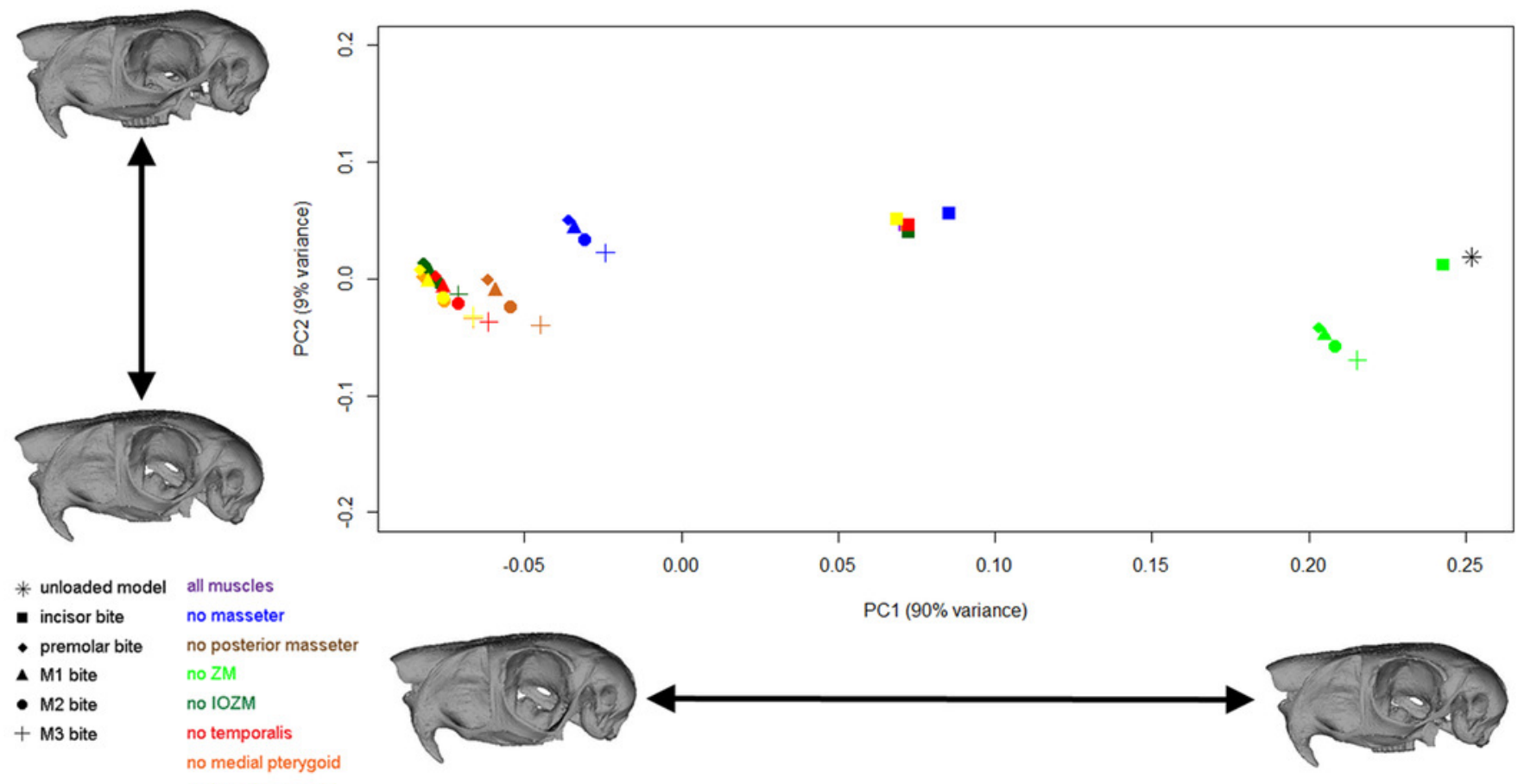

